\title{
Atmospheric Neutrinos Detected with the First KM3NeT Detection Units of ARCA and ORCA
}

\author{
The KM3NeT Collaboration ${ }^{* *}$ \\ \$ https://www.km3net.org/km3net-author-list-for-icrc-2019 \\ E-mail: jannik.hofestaedtefau.de
}

\begin{abstract}
KM3NeT is constructing two next-generation underwater Cherenkov detectors in the deep Mediterranean Sea. The ARCA detector is optimised for TeV-PeV neutrino astronomy, and the smaller and more-densely instrumented ORCA detector is optimised for oscillation research with few-GeV atmospheric neutrinos. The first detection units of both ARCA as well as ORCA have been deployed and are taking data.

In this contribution, an analysis to detect atmospheric neutrinos in the first data of a single detection unit of ORCA and two detection units of ARCA is presented. With ORCA, a sample of 77 neutrino candidates in 125.3 days of livetime is extracted. A good agreement with expectations from Monte-Carlo simulations is found. With ARCA, 6 neutrino candidates are identified in 53.2 days of livetime.
\end{abstract}

Corresponding authors: Rosa Coniglione ${ }^{1}$, Jannik Hofestädt ${ }^{\dagger 2}$, Anna Sinopoulou ${ }^{3}$, Ekaterini Tzamariudaki $^{3}$, Dmitry Zaborov ${ }^{4}$

${ }^{1}$ INFN, Laboratori Nazionali del Sud, Via S. Sofia 62, Catania, 95123 Italy

${ }^{2}$ Erlangen Centre for Astroparticle Physics (ECAP), Friedrich-Alexander-Universität ErlangenNürnberg, Erwin-Rommel-Straße 1, 91058 Erlangen, Germany

${ }^{3}$ NCSR Demokritos, Institute of Nuclear and Particle Physics, Ag. Paraskevi Attikis, Athens, 15310 Greece

${ }^{3}$ A.I. Alikhanov Institute for Theoretical and Experimental Physics of NRC 'Kurchatov Institute', Bolshaya Cheremushkinskaya ulitsa 25, Moscow, 117218 Russia

36th International Cosmic Ray Conference -ICRC2019-

July 24th - August 1st, 2019

Madison, WI, U.S.A.

\footnotetext{
* for collaboration list see PoS(ICRC2019)1177

${ }^{\dagger}$ Speaker.
} 


\section{Introduction}

The KM3NeT Collaboration [1] is currently constructing two deep-sea Cherenkov detectors in the Mediterranean Sea. The ARCA (Astroparticle Research with Cosmics in the Abyss) detector is deployed $80 \mathrm{~km}$ offshore Sicily, Italy, at a depth of $3450 \mathrm{~m}$. ARCA's main objective is the discovery and subsequent observation of $\mathrm{TeV}-\mathrm{PeV}$ astrophysical neutrino sources. The ORCA (Oscillation Research with Cosmics in the Abyss) detector is deployed $40 \mathrm{~km}$ offshore Toulon, France, at a depth of $2450 \mathrm{~m}$. ORCA's main objective is the determination of the neutrino mass ordering by measuring the oscillation probabilities of atmospheric neutrinos with energies of a few $\mathrm{GeV}$.

ARCA and ORCA share the same technology. A main detector component is the digital optical module (DOM), a pressure-resistant glass sphere housing 31 3-inch photo-multiplier tubes (PMTs) and their associated electronics. The DOMs are arranged in string-like structures, called detection units (DUs), anchored to the seabed and held vertically by the buoyancy of the DOMs as well as a buoy. The main difference between the two detector designs is the density of photosensors, which is optimised for the different targeted neutrino energy regimes. The vertical spacing between the DOMs along a DU is $36 \mathrm{~m}$ for ARCA and $9 \mathrm{~m}$ for ORCA. The horizontal spacing between the DUs is $\sim 90 \mathrm{~m}$ for ARCA and $\sim 20 \mathrm{~m}$ for ORCA. In their final configurations, ARCA will consist of 230 DUs instrumenting a volume of $\sim 1 \mathrm{~km}^{3}$ of seawater, and ORCA will consist of 115 DUs instrumenting $\sim 0.008 \mathrm{~km}^{3}$.

The first DU of ARCA was deployed in December 2015, followed by two additional DUs in May 2016. One of these two DUs could not be operated, therefore data with two DUs were taken. Due to electrical problems in the seabed network infrastructure, ARCA detector operation was on hold between April 2017 and January 2019, after which data taking continued with one DU. The first DU of ORCA was installed in September 2017 and operated until December 2017, when a failure of the main electro-optical cable occurred. The deployed DU was recovered for inspection. After replacement of a part in the main cable, another DU (different from the DU deployed in 2017) was deployed in February 2019. Two further DUs of ORCA were deployed in May 2019, however, during this deployment the previously deployed DU was damaged. In the beginning of July 2019 two additional DUs were deployed. At the time these proceedings are written, ORCA is running with four DUs and ARCA with one DU.

In this paper, the first atmospheric neutrinos detected with the deployed KM3NeT detection units of ORCA and ARCA are presented. The neutrino candidates are detected with the first two DUs of ARCA and one DU of ORCA. The detector configuration and the considered data sample are described in Section 2. The Monte-Carlo simulations of the detector response for atmospheric neutrinos and atmospheric muon background are described in Sec. 3. Event reconstruction and neutrino event selection are discussed in Sec. 4 and Sec. 5. The detected atmospheric neutrinos are presented in Sec. 6 and conclusions are drawn in Sec. 7.

\section{Detector configuration and data sample}

Data sample for ARCA2: The considered data taking period for ARCA ranges from December 23, 2016 to March 2, 2017. High-quality data (in terms of detector conditions) are selected with an effective livetime of 53.2 days with both DUs in operation. This detector configuration is referred to as ARCA2. 
Data sample for ORCA1: Data from two different data taking periods are considered, each period with a single DU. The data taking periods are from September 28, 2017 to December 13, 2017 and from March 13, 2019 to May 15, 2019. The DUs operating during these two periods were not the same, but very similar in their configuration and performance, justifying the combination of the two data samples. High-quality data (in terms of detector conditions) are selected with an effective livetime of 123.3 days with a similar share between the two periods. This detector configuration is referred to as ORCAl.

\section{Simulations for detector response to atmospheric neutrinos and muons}

Detailed Monte-Carlo (MC) simulations are used to simulate the detector response to atmospheric muons and neutrinos. The simulation chain used for the analysis presented in this paper is similar to the one described in [1], with one important difference in the last step, where the PMT response and the readout as well as the onshore data filtering is simulated. In contrast to [1], where only sensitivity studies based on simulations were performed, the run-by-run strategy is followed here in order to take into account the time evolution of the data acquisition, in particular the conditions in terms of optical background rates and photon detection efficiencies for each PMT. The PMT photon detection efficiencies are measured and monitored in-situ using the calibration procedure described in [2].

For ORCA, the simulated neutrino and anti-neutrino events are weighted to reproduce the conventional atmospheric neutrino flux following the Honda model [3] and accounting for neutrino oscillations. For ARCA, that addresses higher neutrino energies, oscillation effects are neglected and in addition to the Honda model a prompt component and a knee correction is added to the flux as described in [1]. The simulated event statistics of atmospheric muons corresponds to the livetime of the considered data sample for ARCA2 and ORCA1.

\section{Event Reconstruction}

The collected data are analysed using the standard muon track reconstruction software [4]. The reconstruction chain includes a full-sky grid scan with a prefit, followed by a simple $\chi^{2}$-fit and a full maximum-likelihood fit. The key ingredient for event reconstruction is the large scattering length of deep-sea water. Most of the Cherenkov light is detected with a small time residual $t_{\text {res }}$ between the measured and expected photon arrival time. In a final step of the reconstruction, the observed start position of the muon trajectory is determined by back-projecting the hits onto the track under the Cherenkov angle. The first associated emission point which exceeds the random background level is selected as start position $P_{\text {start }}$.

Event visualisation Example event displays for an up-going neutrino candidate and a downgoing atmospheric muon event recorded with ORCA1 are shown in Fig. 1. The y-axis shows the height in the detector and the $\mathrm{x}$-axis shows the measured hit time. The signal hit pattern can easily be distinguished from the random optical background noise of about $5-10 \mathrm{kHz}$ per PMT, which results in about 5-10 noise hits on all $18 \times 31$ PMTs of the entire DU for the shown time window of $1500 \mathrm{~ns}$. The propagation pattern of a down-going atmospheric muon event (Fig. 1 right) can easily be distinguished from the one for up-going neutrino-induced events (Fig. 1 left). A neutrino 
candidate recorded on both DUs of ARCA2 is shown in Fig. 2. Both Fig. 1 (left) and Fig. 2 show neutrino candidates fulfilling the event selection criteria, described below.

event=1668, run=2974, \#hits=26, cos(zenith_reco)=-0.469 DU 2

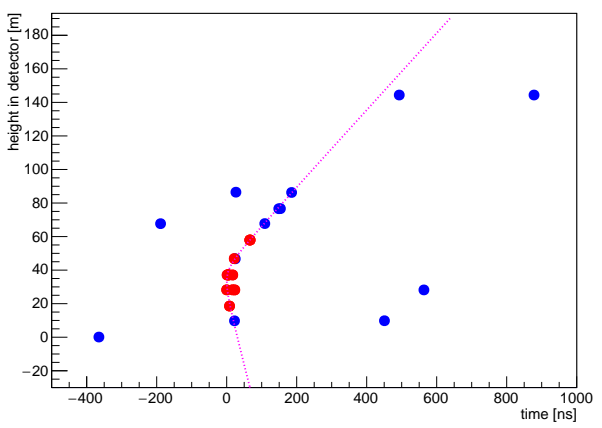

event=9693, run=2974, \#hits=126, $\cos ($ zenith_reco) $=0.997$ DU 2

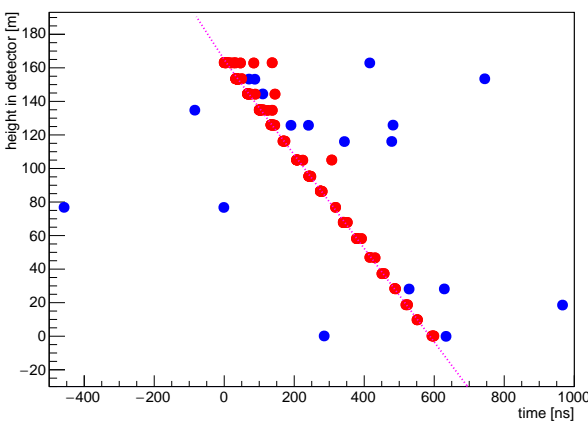

Figure 1: Height in the detector versus the time of the recorded PMT hits with ORCA1 from a neutrino candidate (left) reconstructed as up-going with $\cos ($ zenith $) \approx-0.47$ and a vertically down-going muon (right). Height is given with respect to the bottommost DOM. Hits used in the trigger are shown as red points and all other hits as blue points. The dotted line indicates the expected photon arrival time from the reconstructed muon track. The turning point of the line (left plot) corresponds to the point of closest approach of the muon track to the DU.

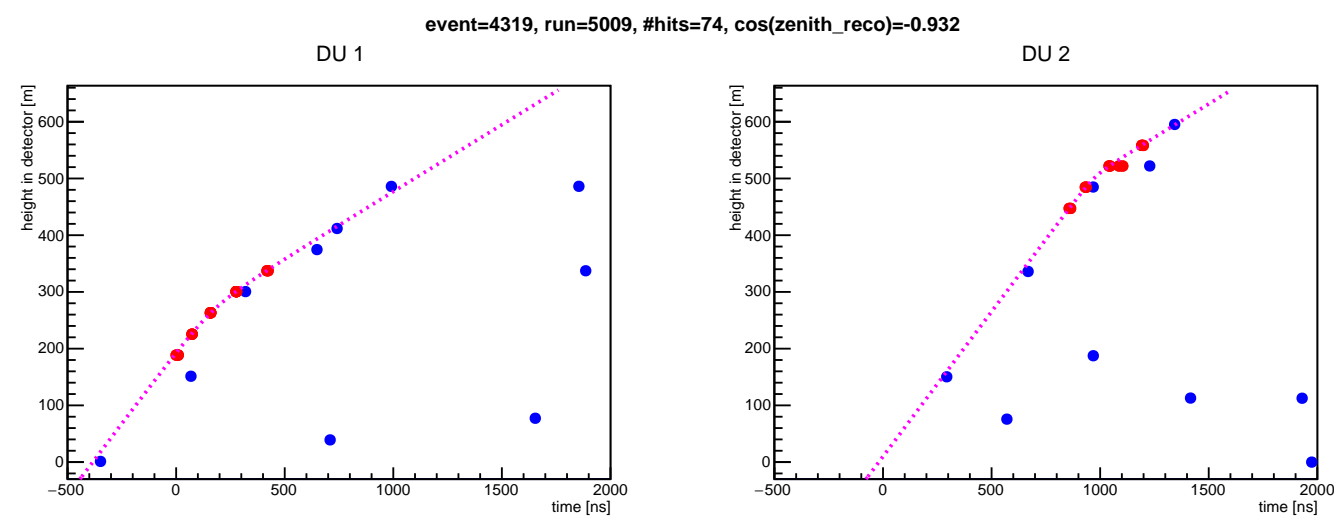

Figure 2: Height in the detector versus the time of the recorded PMT hits seen in both DUs of ARCA2 for a neutrino candidate reconstructed as up-going.

\section{Atmospheric muon rejection and neutrino event selection}

The principal background for identifying neutrino events is caused by atmospheric muons, including single muons as well as muon bundles. The rate of recorded atmospheric muon events is about $\sim 2 \mathrm{~Hz}$ for ORCA1 and $\sim 0.2 \mathrm{~Hz}$ for ARCA2, while only $\sim 10$ atmospheric neutrinos per day are recorded with ORCA1 and $\sim 1$ with ARCA2. Atmospheric muons always travel in a downward direction but can be sometimes wrongly reconstructed as up-going, particularly when only a truncated view of the event is available with only a single DU or two DUs in the case of ORCA1 and ARCA2, respectively. 
Atmospheric muon are rejection works as follows: Events reconstructed as down-going are excluded with a simple cut on the reconstructed zenith angle. Next, events reconstructed as upgoing with a bad fit quality are excluded using cuts on the fit quality. Finally, atmospheric muon events reconstructed as up-going with a good fit quality are suppressed by a set of dedicated selection criteria. The latter event category is relatively important in the present study due to the small size of the detectors (e.g. ORCA1 is a one-dimensional detector). The exact definitions of the quantities used for neutrino event selection as well as the cut values are different for ARCA2 and ORCA1 due to the different instrumentation densities of the two detectors, resulting in a different strategy to identify neutrino events.

Some neutrino event selection criteria are explained and illustrated below for the case of ORCA1. The main categories are:

- zenith angle: events reconstructed as up-going are selected.

- event reconstruction quality: likelihood $L$ value of the maximum-likelihood fit as well as the relative likelihood value per hit used in the fit, $L / N_{\text {hits }}$.

- up/down ambiguity: events which allow for down-going reconstruction solution are considered ambiguous and are excluded.

- topology: events which show little activity downstream, i.e. above for up-going events, from the point of closest approach are considered potentially ambiguous and are excluded.

- number of signal-like hits: signal-like hits have a small time residual $t_{\text {res }}$ and are recorded by nearby DOMs with a not too large photon travel distance from the expected Cherenkov photon emission position along the muon track to the PMT.

- hit distributions: based on temporal and spacial distribution of signal-like hits.

- containment: based on the location of the reconstructed start position $P_{\text {start }}$ of the track relative to the detector (further discussed below).

Data-MC comparison for ORCA1 After applying all event selection criteria except the containment condition, the main class of atmospheric muon events passing this selection are close-to horizontal muons that go below the detector. These events produce an up-going Cherenkov light front in the detector and are therefore often mis-reconstructed as up-going. A very effective rejection of these events can be achieved using the reconstructed start position of the track $P_{\text {start }}$.

Fig. 3 shows the distribution of reconstructed start positions for atmospheric muons (left) and atmospheric neutrinos (right). Atmospheric muon events cluster at the height of the bottommost DOM, while atmospheric neutrino events are distributed over the entire vertical position along the DU. The distribution in data (black diamond markers) is in agreement with MC expectations. Based on the simulation expectations for atmospheric muons and neutrinos, a containment volume (red dashed line) is defined. This allows an efficient rejection of the atmospheric muon background.

Fig. 4 (left) shows the distribution of $L / N_{\text {hits }}$ measured in data and expected from atmospheric muon and neutrino simulations. The containment criterion is not applied, i.e. also those events outside the containment volume in Fig. 3 are shown. A good data/MC agreement is achieved, also 
in the region of small $L / N_{\text {hits }}$, where mis-reconstructed atmospheric muon events dominate. The distribution of the number of DOMs with signal-like hits after the final event selection shown in Fig. 4 (right) is also well described by the MC simulations.
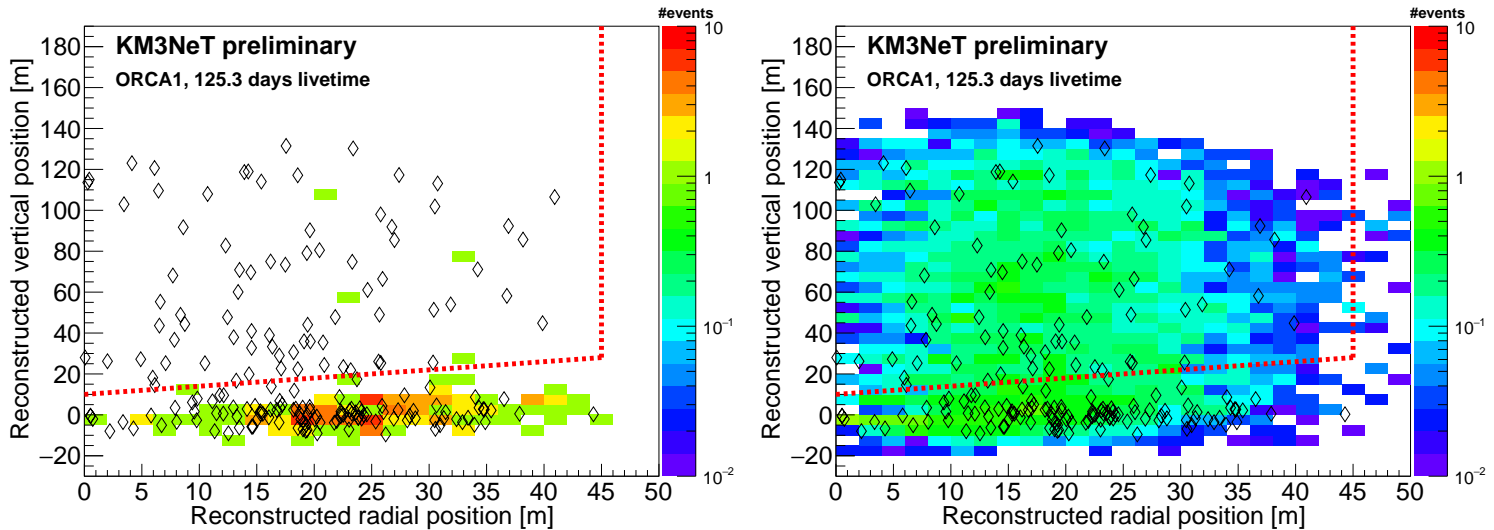

Figure 3: Distribution of reconstructed start positions $P_{\text {start }}$ in ORCA1 as a function of the radial distance to the DU and the vertical position along the DU for atmospheric muons (left) and atmospheric neutrinos (right) from MC simulations (coloured). The black diamond markers show the observed $P_{\text {start }}$ in data. All event selection criteria except the containment criterion are applied. The containment criterion is indicated as a red dashed line.
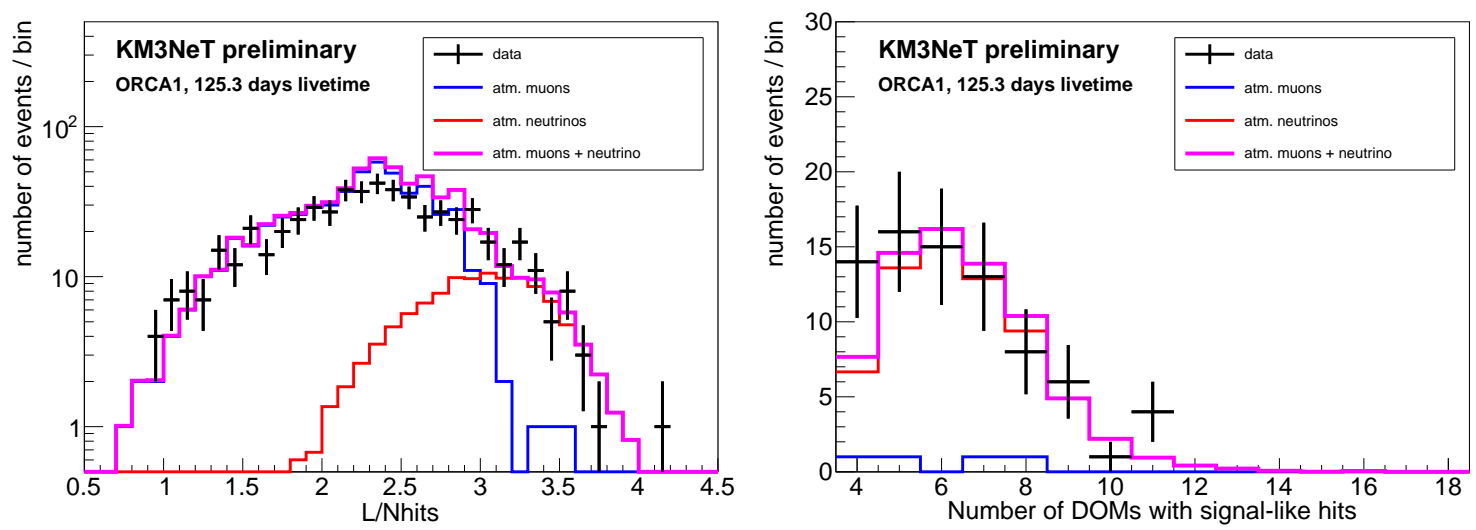

Figure 4: Left: distribution of $L / N_{\text {hits }}$ at an intermediate level of the event selection (see text). Data (black) is compared with expectations from atmospheric muon (blue), neutrino simulations (red) and their combination (magenta). Events are selected with $L / N_{\text {hits }}>2.5$. Right: distribution of the number of DOMs with signallike hits (small time residual) a the final event selection requirements are applied.

\section{Detected neutrinos}

ORCA1: 77 neutrino candidates reconstructed as up-going are observed in the considered data sample of 125.3 days of effective livetime, while 67.5 events are expected from atmospheric neutrino simulations and 4 events from atmospheric muon simulations. Fig. 5 (left) shows the reconstructed zenith-angle distribution. The measured distribution in data is in good agreement with expectations from MC simulations. The neutrino energy spectrum of the simulated neutrinos passing the event selection requirements is shown in Fig 5 (right). The neutrino detection threshold is 
a few GeV. Most of the events are muon (anti-)neutrinos with an energy of a few tens of GeV. In

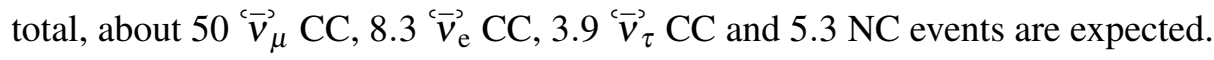
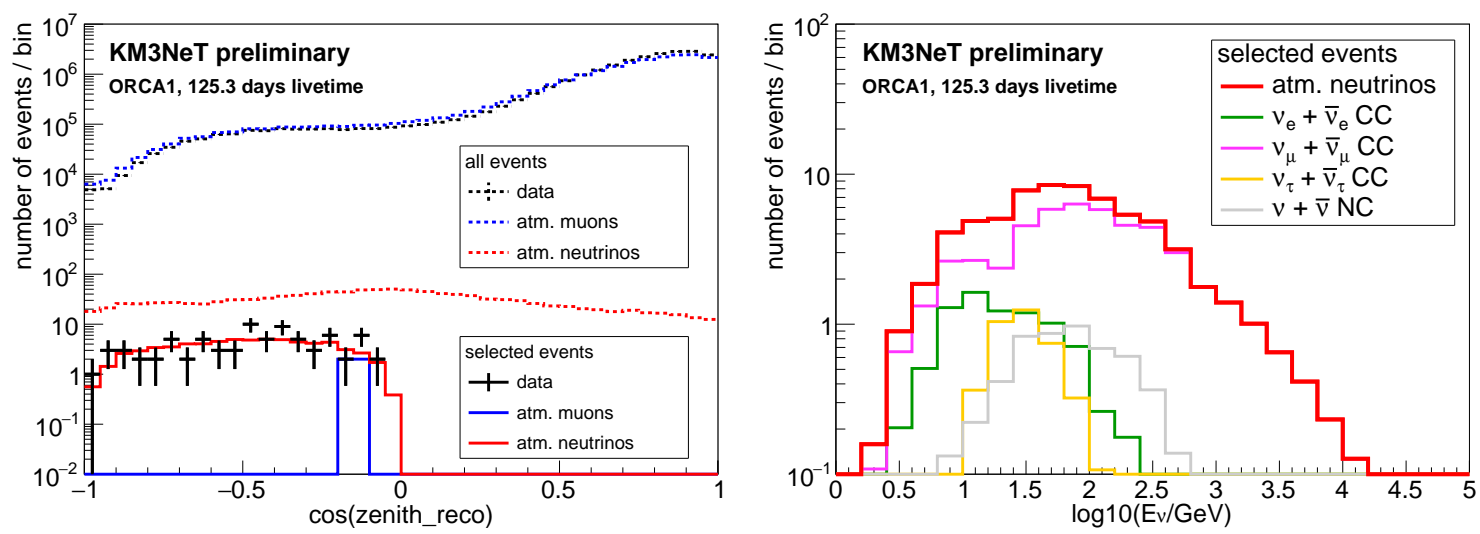

Figure 5: Left: distribution of the cosine of the reconstructed zenith angle in ORCA1 data (back markers), atmospheric neutrino MC (red) and atmospheric muon MC (blue) after applying the event selection requirements (solid lines) and for all events without any event selection (dashed lines). Right: distribution of true neutrino energy $E_{v}$ for events expected to fulfil the event selection criteria for ORCA1. Different colours represent the different neutrino flavours.

ARCA2: 6 neutrino candidates with reconstructed $\cos ($ zenith $)<-0.8$ are found in the considered data sample of 53.3 days of effective livetime, while 3.3 events are expected from atmospheric neutrino simulations and no event from atmospheric muon simulations. Fig. 6 (left) shows the reconstructed zenith-angle distribution with the final event selection applied. The neutrino energy spectrum of the simulated neutrinos passing the event selection requirements is shown in Fig 6 (right). The neutrino detection threshold is about hundred $\mathrm{GeV}$. The selected neutrino events are mainly muon (anti-)neutrinos with typical energies of few hundreds of $\mathrm{GeV}$.
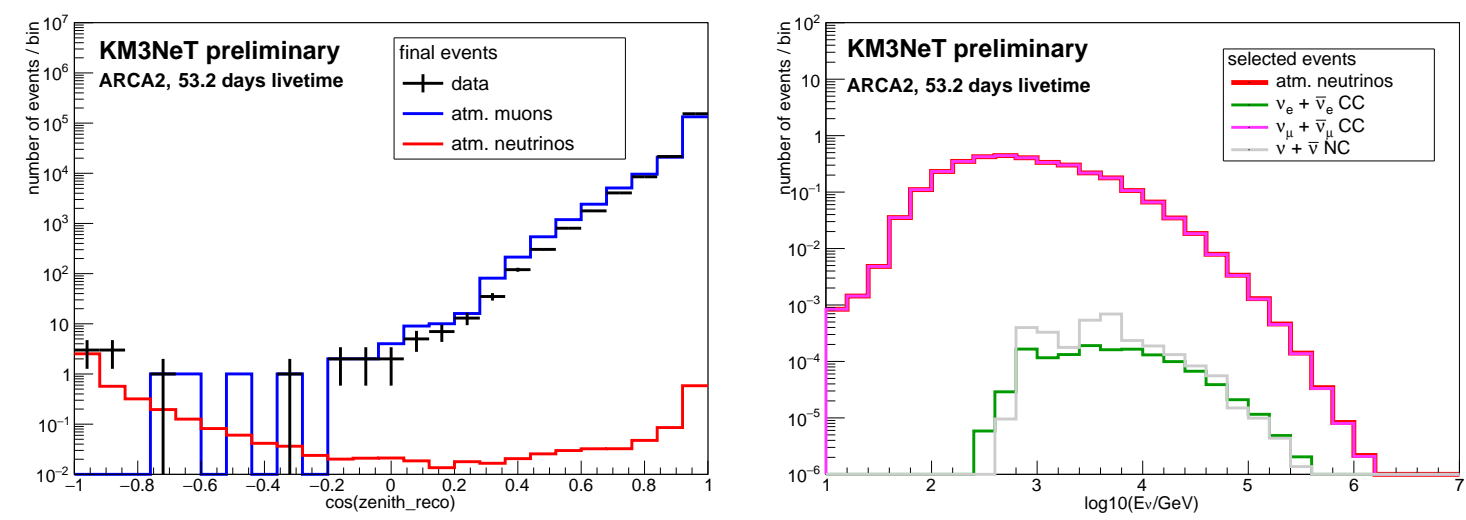

Figure 6: Left: distribution of the cosine of the reconstructed zenith angle in ARCA2 data (black markers), atmospheric neutrino MC (red) and atmospheric muon MC (blue) after applying the event selection. Right: distribution of true neutrino energy $E_{V}$ for events expected to fulfil the event selection criteria for ARCA2 and reconstructed as $\cos ($ zenith $)<-0.8$. Different colours represent the different neutrino flavours. 


\section{Conclusion and outlook}

With a single DU of ORCA, a clean sample of atmospheric neutrinos is extracted with 77 neutrino candidates in 125.3 days of effective livetime. The contamination of atmospheric muons is estimated to below 10\% The data is in good agreement with expectations from detailed MC simulations, which take into account the detector conditions during data taking.

With two DUs of ARCA, the search for atmospheric neutrinos is more challenging due to the higher neutrino energy detection threshold and the smaller effective livetime analysed, resulting in a significantly lower rate of detectable neutrinos. 6 neutrino candidates have been identified in 53.2 days of effective livetime.

The detection of atmospheric neutrinos already with $\sim 1 \%$ of the full detectors, together with the good data/MC agreement verifies the KM3NeT technology, the detector understanding and detector calibration. This illustrates the capability of the future KM3NeT detectors.

The analysis of the first two-DU and four-DU ORCA data is ongoing. A neutrino candidate recorded on both DUs of ORCA2, deployed in May 2019, is shown in Fig. 7. First results of neutrino oscillation measurements will be possible with the first few DUs of ORCA [5].

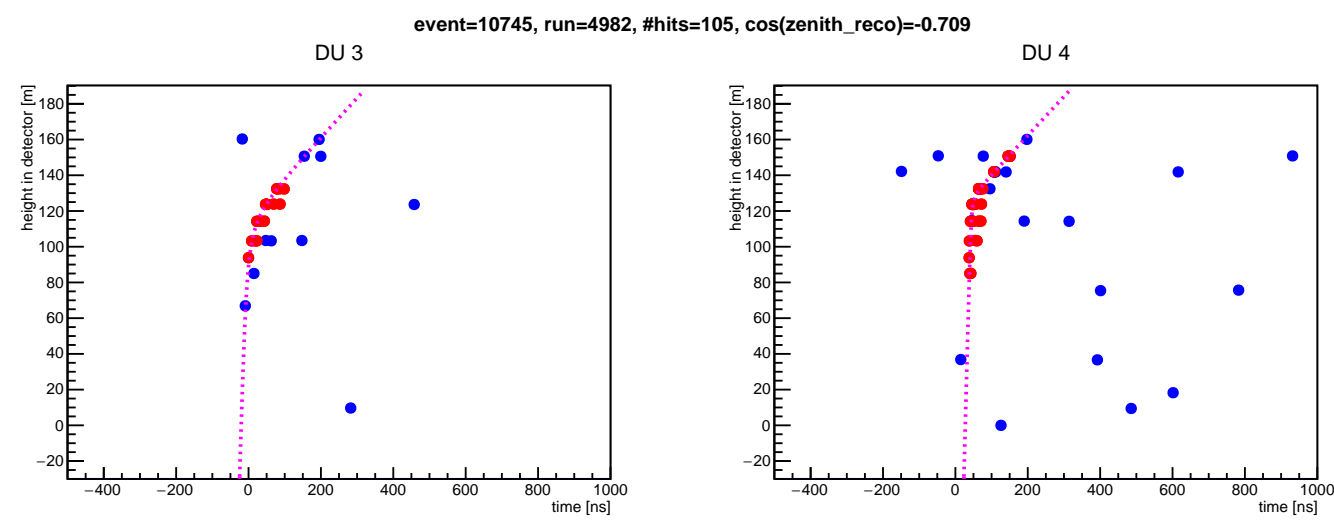

Figure 7: Height in the detector versus the time of the recorded PMT hits seen in both DUs of ORCA2 for a neutrino candidate reconstructed as up-going.

Acknowledgements JH gratefully acknowledges the support by the DFG (grant EB 569/1-1).

\section{References}

[1] S. Adrián-Martínez et al. (KM3NeT Collaboration), 2016 J. Phys. G 43084001

[2] M. Ageron et al. (KM3NeT Collaboration), arXiv:1906.02704[physics.ins-det]

[3] M. Honda et al., Phys. Rev. D 92, 023004 (2015)

[4] K. Melis, A. Heijboer and M. de Jong (for the KM3NeT Collaboration), PoS(ICRC2017)950

[5] B. Strandberg and S. Hallmann (for the KM3NeT Collaboration), PoS(ICRC2019)1019 